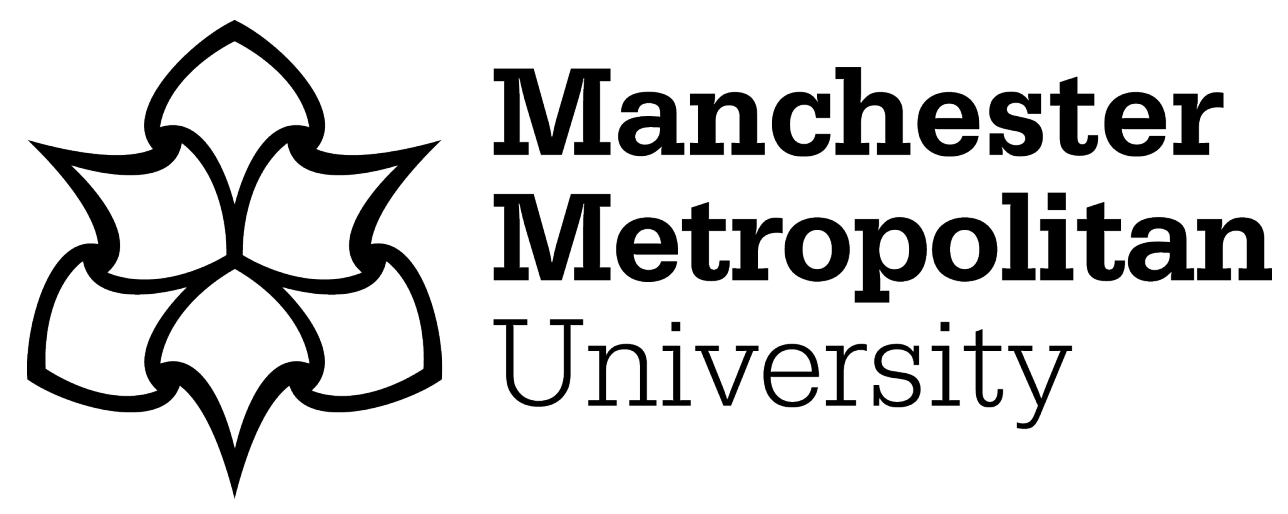

Cuthbert, M, Thomas, C, DosSantos, T and Jones, PA (2019) Application of Change of Direction Deficit to Evaluate Cutting Ability. Journal of Strength and Conditioning Research, 33 (8). pp. 2138-2144. ISSN 1064-8011

Downloaded from: https://e-space.mmu.ac.uk/626029/ Version: Accepted Version

Publisher: National Strength and Conditioning Association

DOI: https://doi.org/10.1519/JSC.0000000000002346

Please cite the published version 


\section{The application of change of direction deficit to evaluate cutting ability.}

Matthew Cuthbert, Christopher Thomas, Thomas Dos'Santos, and Paul A. Jones*.

Directorate of Sport, Exercise \& Physiotherapy, University of Salford, UK.

Corresponding Author: Dr. Paul A. Jones. Directorate of Sport, Exercise and Physiotherapy, University of Salford, Allerton Building, Frederick Road Campus, Salford, Greater Manchester, United Kingdom, M6 6PU.

Tel: (+44) 161295 2371. Email: P.A.Jones@ salford.ac.uk

Running Head: Assessment of cutting ability

No funding was received in support of this work. 


\section{ABSTRACT}

The purpose of this study was to examine the application of the change of direction deficit (CODD) to a $90^{\circ}$ cut test in order to examine whether CODD provides a unique evaluation of an individual's cutting ability. Thirty-six male collegiate team-sport (23 Rugby/ 13 Soccer) athletes (age: $20 \pm 1.4$ years; height: $1.80 \pm 0.08 \mathrm{~m}$; mass: $83 \pm 13.2 \mathrm{~kg}$ ) participated in the study. Each athlete performed 3 trials of a $20 \mathrm{~m}$ sprint (with $5 \mathrm{~m}$ and $10 \mathrm{~m}$ splits) and 2 change of direction [COD] tests $\left(90^{\circ}\right.$ cut and 505 tests) cutting/ turning from both limbs. Completion times for all sprint and COD tests were measured using timing cells. For both COD tests, CODD was determined (COD completion time $-10 \mathrm{~m}$ sprint time). Pearson's correlation was used to explore relationships between sprint times and CODD and completion times. Significant $(\mathrm{P}<0.001)$ moderate to large $(r \geq 0.467)$ correlations between sprint times and $90^{\circ}$ cut completion times for left and right cuts were observed. Non-significant $(\mathrm{P}>0.05)$ trivial to small correlations $(\mathrm{r} \leq 0.199)$ were found between sprint variables and $90^{\circ}$ cut CODD. Significant $(\mathrm{P}<0.001)$ large to very large correlations $(\mathrm{r} \geq 0.531)$ were revealed between left and right $90^{\circ}$ cut and $90^{\circ}$ cut CODD. The results suggest the CODD could be applied to isolate and assess cutting ability in COD speed tests that involve a single cutting maneuver. Failure to inspect CODD could lead to incorrect evaluation of an athletes cutting or COD ability.

Keywords: Agility; change of direction speed; speed; 505 test 


\section{INTRODUCTION}

Development of change of direction (COD) speed is important to provide the physical and technical foundation to develop agility (12). Due to this importance, sports science practitioners need to select an appropriate test to evaluate COD speed. There are a large variety of tests available to practitioners and researchers to assess this quality including; the $505(1,4,10)$, pro-agility (16), L-run $(5,16)$, T-test (11) and Illinois test (19). However, a limitation of many of these tests is their duration. The emphasis an assessment has on COD speed declines the longer the test, becoming more reliant on anaerobic capacity and sprint ability, as more time is spent running between COD actions (12). For instance, typical test durations for the T-test, Illinois, L-Run and Pro-Agility are 8-12 seconds $(11,19), 14-18$ seconds (19), 6-8 seconds $(5,16)$ and 4-5 seconds $(13,16)$, respectively. Therefore, performance on all of these tests may be influenced by metabolic limitations (19) and sprint ability (12) and less on COD ability.

Typical completion times for the 505 test are 2-3 seconds, and thus may potentially avoid the limitation of test duration $(5,10,12)$. The 505 removes much of the task complexity of other tests as with only one turn involved it provides a measure of an individual's ability to negotiate a $180^{\circ}$ turn. However, the completion time of a 505 test may not necessarily provide a measure of COD ability. Nimphius et al. (13) found that only $31 \%$ of the time during a 505 test is spent turning, with the remainder of the time decelerating and accelerating. Therefore, linear sprinting ability may also influence 505 completion times. Several studies have found a relationship between linear sprinting speed and 505 test performance $(5,10,12,14)$, despite the acknowledgement that speed and COD speed are different physical qualities (20). Furthermore, Sayers et al. (14), using 3D motion analysis to examine 505 performance times over distances $0.3,0.5$ and $1 \mathrm{~m}$ before and after the turn (measured as the time for the center of mass to cover each distance before and after the turn), 
revealed strong relationships between 505 time and 5, 10 and $20 \mathrm{~m}$ sprint performance. However, the strength of these relationships reduced when COD ability was measured $0.5 \mathrm{~m}$ and $0.3 \mathrm{~m}$ before and after the turn, highlighting that 505 performance time could be biased by linear sprinting ability.

An innovative approach to overcome this limitation is to apply the COD deficit (CODD) (12,13), whereby a $10 \mathrm{~m}$ sprint time is subtracted from the 505 time. The lower the value, the greater the COD ability. This concept was initially proposed and investigated in 66 collegiate American football players by Nimphius et al. (13), whereby a 10 yard sprint split time was subtracted from a 10 yard split time during a pro-agility test (the resultant time provided an indication of the time taken to negotiate a $180^{\circ}$ turn $)$. Significant $(\mathrm{P}<0.001)$ correlations were observed between pro-agility scores (total and split) time and 10 yard sprint performance. However, a low non-significant correlation was observed between CODD and 10 yard sprint time $(\mathrm{r}=0.19)$, but significant moderate correlations were observed to proagility $(r=0.54)$ and pro-agility split times $(r=0.61)$. This data suggested that the CODD offered a measure of COD speed independent of linear sprinting speed. More recently, Nimphius et al. (12) investigated the application of the COD deficit within the 505 test in 17 cricketers. The authors found that CODD strongly correlated to $505(\mathrm{r}=0.74-0.81)$, but not 10 m sprint time $(r=-0.11-0.10)$, whilst 505 time correlated with sprint time $(r=0.52-0.70)$. Furthermore, when $\mathrm{Z}$ scores were examined, 5 of the 17 subjects were classified differently in terms of COD ability when using 505 or CODD. The results provided further support for the use of CODD to quantify an individual's COD ability, rather than being confounded by linear sprinting speed. Although, it should be noted that $180^{\circ}$ turns are prevalent in cricket and thus, it is unknown whether such findings apply to sports performers where $180^{\circ}$ turns are less utilized (i.e., American football, basketball, soccer and rugby league). Moreover, the authors suggested that further research is required applying the CODD to different COD tasks of 
different angles, particularly for sports where cutting $45^{\circ}$ to $90^{\circ}$ might be more prevalent $(3,7)$.

A test to evaluate cutting ability using a single cut maneuver might be more useful for certain sports, such as rugby union (7) and soccer, where the majority of CODs are reported to be between 0 and $90^{\circ}$ (3). However, again such a test would also be influenced by linear running speed. Therefore, the aim of this study was to examine the application of the CODD principle to a $90^{\circ}$ cut test, to see if the CODD provides a unique evaluation of an individual's cutting ability. It was hypothesized that there would be a strong relationship between linear sprint times and $90^{\circ}$ cut completion times and between $90^{\circ}$ cut completion times and CODD during the $90^{\circ}$ cut test. It is further hypothesized that there would be no relationship between linear sprint times and $90^{\circ}$ cut CODD.

\section{METHODS}

\section{EXPERIMENTAL APPROACH TO THE PROBLEM}

This study used a cross-sectional design where 36 subjects performed three $20 \mathrm{~m}$ sprints with 5, 10 and $20 \mathrm{~m}$ sprint times recorded. All subjects also performed 2 COD tests; a 505 test and a $90^{\circ}$ cut test ( 3 trials each turning from right and left limbs). Completion times were recorded along with CODD (COD test completion time - $10 \mathrm{~m}$ linear sprint time) for both COD tests. To test the study hypothesis Pearson's correlations (normally distributed data) were used to explore relationships between linear sprints times and COD completion times and CODD. Furthermore, for the $90^{\circ}$ cut test, $\mathrm{Z}$ scores were used to evaluate each subject within the sample using both test completion time and CODD, to examine whether subjects were classified differently using either measure. 


\section{SUBJECTS}

36 male collegiate team-sport athletes ( 23 rugby union/league and 13 soccer players) aged between 18 and 22 years (height: $1.80 \pm 0.08 \mathrm{~m}$; mass $83 \pm 13.2 \mathrm{~kg}$ ) participated in the study. For inclusion in the study, all players needed to have played soccer or rugby for a minimum of 5 years and regularly performed 1 game and 2 structured skill based sessions per week. All players were free from injury during the course of the study and none of the player's had suffered prior traumatic knee injury such as anterior cruciate ligament injury. Data collection took place during the player's pre-season. The study was approved by the University's Ethics committee and all subjects were informed of the benefits and risks of the investigation prior to signing an institutionally approved informed consent document to participate in the study.

\section{PROCEDURES}

Each subject attended the lab on one occasion and performed a sprint assessment, $90^{\circ}$ cut test (Figure 1) and 505 test (Figure 2). Each subject was familiar with the test protocols. Each subject attended the lab in a fed and hydrated state, with no caffeine or food intake within 2 hours of data collection. Each subject also performed no training or vigorous exercise within 24 hours of the data collection session. Prior to data collection, each subject performed a typical pre-game warm-up routine incorporating low-intensity jogging (10 minutes) sprint and low intensity plyometric drills (i.e., high knee marching, running, skipping over 20 meters), short sprint $(20 \mathrm{~m})$ and change of direction drills $\left(90^{\circ}\right.$ cuts and $180^{\circ}$ turns), increasing intensity of each effort (e.g. 50\%, $75 \%$ and 100\%).

Sprint Assessment (5m, 10m \& 20m Sprint).

20m sprint times were recorded using Brower (single beam) timing gates (model number BRO001; Brower, Draper, UT, USA) placed at a height of $1 \mathrm{~m}$ (approximately hip height), 
positioned $0 \mathrm{~m}, 5 \mathrm{~m}, 10 \mathrm{~m}$ and $20 \mathrm{~m}$ down an indoor running track (Mondo, SportsFlex, 10 mm; Mondo America Inc., Mondo, Summit, NJ, USA). Players were instructed to stand 0.5 $\mathrm{m}$ behind the first gate, preventing early triggering, in a two-point staggered stance. Players performed two warm up trials at $50 \%$ and $75 \%$ maximum effort before being instructed to give a maximal effort for the whole $20 \mathrm{~m}$. Three trials were completed with one minute's rest between each trial.

Change of Direction Speed Assessment

$90^{\circ}$ cut test

Brower timing gates were placed at a starting point $5 \mathrm{~m}$ away from a marked turning point with two sets placed a further $5 \mathrm{~m}$ away at an angle of $90^{\circ}$ (Figure 1). Subjects again started $0.5 \mathrm{~m}$ away from the first gate, and were instructed to sprint maximally towards the turning point. At the turning point, subjects performed the $90^{\circ}$ cut (by 'planting' the designated leg on the marked turning point and 'cutting' to the opposite side) before sprinting through the second set of timing gates set up on the left or right side, depending on which leg was designated to act as the 'plant' leg for the cut. Right and left $90^{\circ}$ cut performance was defined by the leg in which the subjects used to turn (the 'plant' leg). The trials were carried out turning on each leg for 3 trials and the time in seconds was recorded. The average of all trials was determined and used for further analysis. Two minutes' rest between each trial was given to the athletes.

\section{$<<$ INSERT FIGURE 1 HERE $>>$}

505 test 
For comparison purposes, each subject performed 3 trials each of the 505 test performed turning ('plant leg') with the left and right legs. Brower timing gates were placed at approximately hip height $(1 \mathrm{~m}), 5 \mathrm{~m}$ from a marked turning point (Figure 2). The 505 test started $15 \mathrm{~m}$ away from the turning point (Figure 2). Instructions were given to accelerate as quickly as possible to the turning point (passing the timing gates after $10 \mathrm{~m}$ ), turn $180^{\circ}$ on the left or right leg and sprinting back through the timing gates. Players had two minutes' rest between each trial. Each player performed 3 trials on the left leg and 3 trials on the right leg in a randomized order. The average of all 3 trials on each leg was determined and used for further analysis. For both the $90^{\circ}$ cut and 505 test, CODD was determined using the formula; average COD task completion time - average $10 \mathrm{~m}$ sprint time. The average COD task completion time referred to either the 505 test time or $90^{\circ}$ cut test time, so that CODD was calculated for each task turning or cutting with right and left limb (4 different measures).

\section{<<INSERT FIGURE 2 HERE >>}

\section{STATISTICAL ANALYSES}

Within session reliability for sprint and COD performance was assessed using intraclass correlation coefficients (ICC) and coefficient of variation (\%CV). Minimum acceptable reliability was determined with an ICC >0.7 and CV <15 (2). Statistical analysis was carried out using SSPS software (version 23.0, SPSS, Inc., IL, USA). Normality of all data was confirmed using a Shapiro-Wilks test. Therefore, Pearson's correlation (r) and co-efficients of determination $\left(r^{2} \times 100\right)$ were used to explore relationships between sprint $(5,10$ and 20 m) and COD assessments (90 cut, 505 test and corresponding CODD values). All P-values were Bonferroni adjusted to control for Type 1 error. Statistical significance was set at $\mathrm{P}<$ 0.05. Correlations were evaluated as follows: trivial $(0.0-0.09)$, small $(0.10-0.29)$, moderate $(0.30-0.49)$, large $(0.50-0.69)$, very large $(0.70-0.89)$, nearly perfect $(0.90-0.99)$, and 
perfect (1.0) (9). In addition, using a similar approach to Nimphius et al. (12) for the $90^{\circ}$ cut test, Z scores were determined [(athlete's score - group mean)/group SD] for $90^{\circ}$ cut completion times and $90^{\circ}$ CODD to identify whether athletes were classified differently when using the CODD.

\section{RESULTS}

Descriptives for each variable are presented in Table 1. Low within session coefficient of variation was reported for all sprint and COD tests, whilst acceptable ICC's were reported for all tests with the exception of $5 \mathrm{~m}$ sprint time and 505 right $(5 \mathrm{~m}$; ICC $=0.68, \mathrm{CV}$ $=2.77 \% ; 10 \mathrm{~m} ; \mathrm{ICC}=0.85 ; \mathrm{CV}=2.17 \% ; 20 \mathrm{~m} ; \mathrm{ICC}=0.86 ; \mathrm{CV}=1.65 \% ; 505$ left $; \mathrm{ICC}=$ $0.72 ; \mathrm{CV}=2.76 \% ; 505$ right $; \mathrm{ICC}=0.63 ; \mathrm{CV}=3.10 \% ; 90^{\circ}$ cut left; $\mathrm{ICC}=0.77 ; \mathrm{CV}=$ $2.92 \% ; 90^{\circ}$ cut right; $\left.\mathrm{ICC}=0.72 ; \mathrm{CV}=3.13 \%\right)$.

\section{**INSERT TABLE 1 HERE**}

Table 2 revealed significant $(P<0.001)$ moderate to large $\left(r \geq 0.47 ; r^{2} \geq 22 \%\right)$ correlations between sprint times and $90^{\circ}$ cut completion times for left and right cuts. However, non-significant $(\mathrm{P}>0.05)$ trivial to small correlations $\left(\mathrm{r} \leq 0.199 ; \mathrm{r}^{2} \leq 3.96 \%\right)$ were found between sprint variables and $90^{\circ}$ cut CODD (Table 2). Significant $(\mathrm{P}<0.001)$ large to very large correlations were revealed between left and right $90^{\circ}$ cut and $90^{\circ}$ cut CODD (Table 2).

\section{**INSERT TABLE 2 HERE**}

Sprint times revealed significant $(\mathrm{P}<0.001)$ moderate to large $\left(\mathrm{r} \geq 0.42 ; \mathrm{r}^{2} \geq 17.6 \%\right)$ correlations to 505 test completion times for left and right turns (Table 3). However, only one significant $(\mathrm{P}<0.05)$ moderate correlation was revealed between $10 \mathrm{~m}$ sprint and right 505 CODD, whereas all other correlations between sprint and 505 CODD were trivial to small 
(Table 3). Significant $(\mathrm{P}<0.001)$ moderate to large correlations were revealed between left and right 505 times and 505 CODD (Table 3).

\section{**INSERT TABLE 3 HERE**}

Figure 3 shows z-scores for $90^{\circ}$ cut completion times and $90^{\circ}$ cut CODD, in which large changes in $\mathrm{z}$ scores can be seen between the two measures for each subject. For the right leg cuts, 12 of the 36 subjects were re-classified based on COD ability using the CODD (i.e., the $\mathrm{Z}$ score shifted from positive (slower than average) to negative (faster than average) or vice versa). Similarly for left leg cuts, 8 out of 36 players were re-classified in a similar way.

**INSERT FIGURE 3 HERE**

\section{DISCUSSION}

The aim of the present study was to examine the application of the CODD to a $90^{\circ}$ cut task, to see if the CODD deficit provided a different evaluation of an individual's cutting ability. The main findings of the study were that significant $(\mathrm{P}<0.001)$ moderate to large $(\mathrm{r} \geq$ $\left.0.47 ; r^{2} \geq 22 \%\right)$ correlations were observed between sprint times $(5,10$ and $20 \mathrm{~m})$ and $90^{\circ}$ cut completion times for left and right cuts. However, when using the $90^{\circ}$ cut CODD, nonsignificant $(P>0.05)$ trivial to small correlations $\left(r \leq 0.20 ; r^{2} \leq 4 \%\right)$ were found between sprint variables and $90^{\circ}$ cut $\mathrm{CODD}$, with significant $(\mathrm{P}<0.001)$ large to very large association between left and right $90^{\circ}$ cut and $90^{\circ}$ cut CODD remaining. 505 test completion times revealed significant $(\mathrm{P}<0.001)$ moderate to large $\left(\mathrm{r} \geq 0.42 ; \mathrm{r}^{2} \geq 17.6 \%\right)$ correlations to sprint times. However, when using CODD with the exception of a moderate significant $(\mathrm{P}<$ 0.05) correlation between $10 \mathrm{~m}$ sprint time and right $505 \mathrm{CODD}$, all other correlations between sprint and 505 CODD were trivial to small, whilst significant $(\mathrm{P}<0.001)$ moderate 
to large correlations remained between 505 times and 505 CODD. In addition, when examining calculated individual Z-scores for right leg cuts 12 of the 36 subjects were reclassified for COD ability using the CODD, whereas for left leg cuts, 8 out of 36 players were re-classified based on CODD. The findings substantiated the study hypothesis and that the CODD deficit can be applied to a $90^{\circ}$ cut test to isolate COD ability, rather than be confounded by linear sprinting speed.

The results of present the study support the findings of Nimphius et al. (13) and Nimphius et al. (12) for the 505 test or single $180^{\circ}$ turns, in that 505 test performance was moderately to largely correlated to linear sprint times, but when using the CODD the correlations to linear sprinting ability were small or absent. Furthermore, 505 test performance showed a moderate correlation $(r=0.48)$ to CODD in the 505 test; suggesting that $23 \%$ of the variation in 505 test performance could be explained by COD ability as measured by the CODD. The results of the present study provide further support for the utilization of CODD to quantify COD ability during a 505 test, particularly in sports where the $180^{\circ}$ turn is common such as running between wickets when batting in cricket, or during certain running routes in American football (i.e., a 'stop n go').

An advantage of the present study is that the results show that the CODD can be used to quantify COD ability during a sharp cutting task, which often is more widely observed in field and court sports such as basketball (15), American football (6), netball (18), soccer (3) and rugby union (7). The $90^{\circ}$ cut task used in the present study revealed large correlations to linear sprint performance $(5,10$ and $20 \mathrm{~m})$. When using the CODD, the correlations to linear sprint times were trivial to small $(\mathrm{r} \leq 0.32)$. Whereas large $(\mathrm{r} \geq 0.53)$ correlations were observed between $90^{\circ}$ cut completion times and $90^{\circ}$ cut CODD. These results suggested that applying the CODD to a $90^{\circ}$ cut within a COD speed test isolates 'cutting' ability, and is not influenced by linear running speed, which is the case with the $90^{\circ}$ cut completion times. This 
finding is similar to what was observed with the 505 test in this study and in previous research (9).

The calculated $\mathrm{Z}$ scores (Figure 3) revealed that for the right and left leg turns, 12 and 8 out of 36 athletes, respectively, were classified differently with regard to COD ability when using $90^{\circ}$ cut CODD compared to completion times. These results provided further support for the application of CODD to the $90^{\circ}$ cut task to isolate COD ability. These findings are similar to the findings of Nimphius et al. (12) and suggest that without applying the CODD to such tasks could lead to incorrect training prescription with regard to COD training of athletes. Furthermore, 5 athletes observed similar trends across both legs. However, 7 athletes were classified differently on right leg cuts, but classified the same on left leg cuts, whereas 3 athletes where classified differently for left leg cuts, but the same for right leg cuts. This further highlights the need to assess CODD across both limbs to ensure that training prescription is directed to ensure athletes become equally proficient at cutting from either limb.

Although, low and acceptable CV's were reported $(\leq 3.13 \%)$ for all variables in the present study. Only moderate to acceptable ICC's $(0.63-0.86)$ were found. This lower relative reliability reported may be due to the use of single beam timing cells. The reliability (ICC's) of these variables would be enhanced with the use of dual-beam timing cells (8). Therefore, application of the CODD and the $90^{\circ}$ cut test in future research or applied practice should use dual-beam timing cells to enhance within session reliability of these tests. Further limitations pertain to the University-level male soccer and rugby athletes used in the study. Although, both sports involve high prevalence of cutting actions $(3,7)$, the heterogeneity in subject population may have influenced the magnitude of correlations observed. Future studies should evaluate the application of CODD to individual sports and consider the application of CODD to high level professional athletes. Finally, it was beyond the scope of 
the study to collect additional biomechanical data to evaluate the biomechanical characteristics of faster CODD performances and to gather a greater understanding of how to influence this variable to inform future training prescription. Future research into the technical determinants of CODD is required to inform future practice.

To conclude, the CODD can be applied to a $90^{\circ}$ cut task and thus, could be applied to sports where cutting ability is of high importance. The CODD when applied to a $90^{\circ}$ cut provides an isolated measure of 'cutting' ability, removing the influence of running speed on such tests of COD ability. Future research is required to apply the CODD to cutting tasks in a variety of cutting sports to provide information of how CODD differs between different levels and populations of athlete. Furthermore, an investigation on the technical determinants of CODD in cutting is required to inform coaches with regard to instructing athletes to enhance 'cutting' ability.

\section{PRACTICAL APPLICATIONS}

Sports scientists should apply the CODD to COD speed tests that involve a single cutting movement to isolate and assess the athletes 'cutting' ability from linear running speed. Failure to apply the CODD to such cutting tasks could lead to incorrect evaluation of an athletes cutting (COD) ability and lead to errors in training prescription. The $90^{\circ}$ cut test modified from the 505 test to involve a cutting maneuver is recommended in sports where cutting is highly prevalent. 


\section{REFERENCES}

1. Barber OR, Thomas C, Jones PA, McMahon JJ, and Comfort P. Reliability of the 505 Change-of-direction test in Netball Players. Int J Sports Physiol Perf 11:377-380, 2016.

2. Baumgartner TA, and Chung H. Confidence Limits for Intraclass Reliability Coefficients. Meas Phys Educ Exerc Sci 5:179-88, 2001.

3. Bloomfield J, Polman R, and O'Donoghue P. Physical demands of different positions in FA Premier League soccer. J Sports Sci Med 6: 63-70, 2007.

4. Draper JA, and Lancaster MG. The 505 test: A test for agility in the horizontal plane. Aust J Sci Med Sport 17:15-18, 1985.

5. Gabbett TJ, Kelly JN, and Sheppard JM. Speed, change of direction speed, and reactive agility of rugby league players. J Strength Cond Res. 22: 174-181, 2008.

6. Gleason BH, Kramer JB, and Stone MH. Agility training for American football. Strength Cond J 37: 65-71, 2015.

7. Green BS, Blake C, and Caulfield BM. A comparison of cutting technique performance in rugby union players. J Strength Cond Res 25: 2668-2680, 2011.

8. Haugen, T, and Buchheit, M. Sprint running performance monitoring: Methodological and practical considerations. Sports Med 46: 641-656, 2016.

9. Hopkins WG. Measures of reliability in sports medicine and science. Sports Med 30: $1-15,2000$.

10. Jones $\mathrm{P}$, Bampouras $\mathrm{T}$, and Marrin $\mathrm{K}$. An investigation into the physical determinants of change of direction speed. J Sports Med Phys Fitness 49: 97-104, 2009. 
11. Munro AG, and Herrington LC. Between session reliability of four hop tests and the agility t-test. J Strength Cond Res 25: 1470-1477, 2011.

12. Nimphius S, Callaghan SJ, and Lockie, R. Change of direction deficit: A more isolated measure of change of direction performance than total 505 time. J Strength Cond Res 30: 3024-3032, 2016.

13. Nimphius S, Geib G, Spiteri T, and Carlisle, D. "Change of direction" deficit measurement in division I American football players. J Aust Strength Cond 21: 115117,2013

14. Sayers MGL. Influence of test distance on change of direction speed test results. $J$ Strength Cond Res 29: 2412-2416, 2015.

15. Shimokochi Y, Ide D, Kokubu, M and Nakaoji T. Relationships among performance of lateral cutting maneuver from lateral sliding and hip extension and abduction motions, ground reaction force and body center of mass height. J Strength Cond Res 27: 1851-1860, 2013.

16. Sierer SP, Battaglini CL, Mihalik JP, Shields EW, and Tomasini, NT. The National Football League combine: Performance differences between drafted and non-drafted players entering the 2004-2005 drafts. J Strength Cond Res 22: 6-12, 2008.

17. Sporis G, Jukic I, Milanovic L, and Vucetic V. Reliability and factorial validity of agility tests for soccer players. J Strength Cond Res 24: 679-686, 2010.

18. Sweeting AJ, Aughey RJ, Cormack SJ, and Morgan S. Discovering frequently recurring movement sequences in team-sport athlete spatiotemporal data, J Sports Sci 35: 2439-2445, 2017.

19. Vescovi JD, and McGuigan MR. Relationships between sprinting, agility, and jump ability in female athletes. J Sports Sci 26: 97-107, 2008. 
20. Young WB, McDowell MH, and Scarlett BJ. Specificity of sprint and agility training methods. J Strength Cond Res 15: 315-319, 2001.

\section{LIST OF TABLES AND FIGURES}

Table 1. Descriptives for sprint and COD tests.

Table 2. Relationships between sprint and $90^{\circ}$ cut completion times and $90^{\circ}$ cut CODD.

Table 3. Relationships between sprint and 505 completion times and 505 CODD.

Figure 1. Example of left and right $90^{\circ}$ cutting task.

Figure 2. Example of the 505 COD test

Figure 3. Z -scores for $90^{\circ}$ cut completion times and $90^{\circ}$ cut CODD for left leg cuts (top) and right leg cuts (bottom). 
Table 1. Descriptives for sprint and COD tests.

\begin{tabular}{|l|c|c|c|}
\hline Variable & Mean \pm SD & Right & Left \\
\hline $5 \mathrm{~m}(\mathrm{~s})$ & $1.07 \pm 0.06$ & & \\
\hline $10 \mathrm{~m}(\mathrm{~s})$ & $1.87 \pm 0.13$ & & \\
\hline $20 \mathrm{~m}(\mathrm{~s})$ & $3.19 \pm 0.18$ & & $2.37 \pm 0.21$ \\
\hline $90^{\circ} \mathrm{Cut}(\mathrm{s})$ & $2.34 \pm 0.17$ & $2.34 \pm 0.15$ & $0.49 \pm 0.15$ \\
\hline $90^{\circ} \mathrm{Cut}$ CODD (s) & $0.47 \pm 0.12$ & $0.47 \pm 0.10$ & $2.51 \pm 0.14$ \\
\hline 505 test (s) & $2.50 \pm 0.15$ & $2.48 \pm 0.14$ & $0.64 \pm 0.12$ \\
\hline $505 \mathrm{CODD}(\mathrm{s})$ & $0.63 \pm 0.14$ & $0.60 \pm 0.12$ & \\
\hline
\end{tabular}

Note CODD $=$ Change of direction deficit. 
Table 2 Relationships between sprint and $90^{\circ}$ cut completion times and $90^{\circ}$ cut CODD.

\begin{tabular}{|c|c|c|}
\hline & $\begin{array}{c}90^{\circ} \mathrm{Cut} \\
\mathrm{r}\left(\mathrm{r}^{2}\right)\end{array}$ & $\begin{array}{c}90^{\circ} \text { Cut CODD } \\
r\left(r^{2}\right)\end{array}$ \\
\hline \multicolumn{3}{|l|}{ Left } \\
\hline $5 \mathrm{~m}$ & $0.47(22.1 \%)^{* *}$ & $0.06(0.4 \%)$ \\
\hline $10 \mathrm{~m}$ & $0.61(37.2 \%)^{* *}$ & $0.04(0.2 \%)$ \\
\hline $20 \mathrm{~m}$ & $0.64(41 \%) * *$ & $0.20(4 \%)$ \\
\hline $90^{\circ} \mathrm{cut}$ & - & $0.77(59.3 \%)^{* *}$ \\
\hline \multicolumn{3}{|l|}{ Right } \\
\hline $5 \mathrm{~m}$ & $0.64(41 \%)^{* *}$ & $0.10(1 \%)$ \\
\hline $10 \mathrm{~m}$ & $0.75(56.3 \%)^{* *}$ & $-0.01(0.01 \%)$ \\
\hline $20 \mathrm{~m}$ & $0.84(70.6 \%)^{* *}$ & $0.32(10.2 \%)$ \\
\hline $90^{\circ}$ cut & - & $0.531(28.2 \%)^{* *}$ \\
\hline
\end{tabular}

** $\mathrm{P}<0.001 ; * \mathrm{P}<0.05 . \mathrm{CODD}=$ change of direction deficit. 
Table 3 Relationships between sprint and 505 completion times and 505 CODD.

\begin{tabular}{|c|c|c|}
\hline & $\begin{array}{c}505 \text { test } \\
r\left(r^{2}\right)\end{array}$ & $\begin{array}{c}505 \text { CODD } \\
r\left(r^{2}\right)\end{array}$ \\
\hline \multicolumn{3}{|l|}{ Left } \\
\hline $5 \mathrm{~m}$ & $0.42(17.6 \%)^{*}$ & $-0.21(4.4 \%)$ \\
\hline $10 \mathrm{~m}$ & $0.54(29.2 \%)^{* *}$ & $-0.27(7.3 \%)$ \\
\hline $20 \mathrm{~m}$ & $0.48(23 \%)^{* *}$ & $-0.11(1.2 \%)$ \\
\hline 505 test & - & $0.54(29.2 \%)^{* *}$ \\
\hline \multicolumn{3}{|l|}{ Right } \\
\hline $5 \mathrm{~m}$ & $0.57(32.5 \%)^{* *}$ & $-0.04(0.2 \%)$ \\
\hline $10 \mathrm{~m}$ & $0.49(24 \%)^{* * *}$ & $-0.38(14.4 \%)^{*}$ \\
\hline $20 \mathrm{~m}$ & $0.51(26 \%)^{* *}$ & $-0.19(3.6 \%)$ \\
\hline 505 test & - & $0.48(23 \%)^{* *}$ \\
\hline
\end{tabular}

** $P<0.001 ; * P<0.05$. CODD = change of direction deficit. 


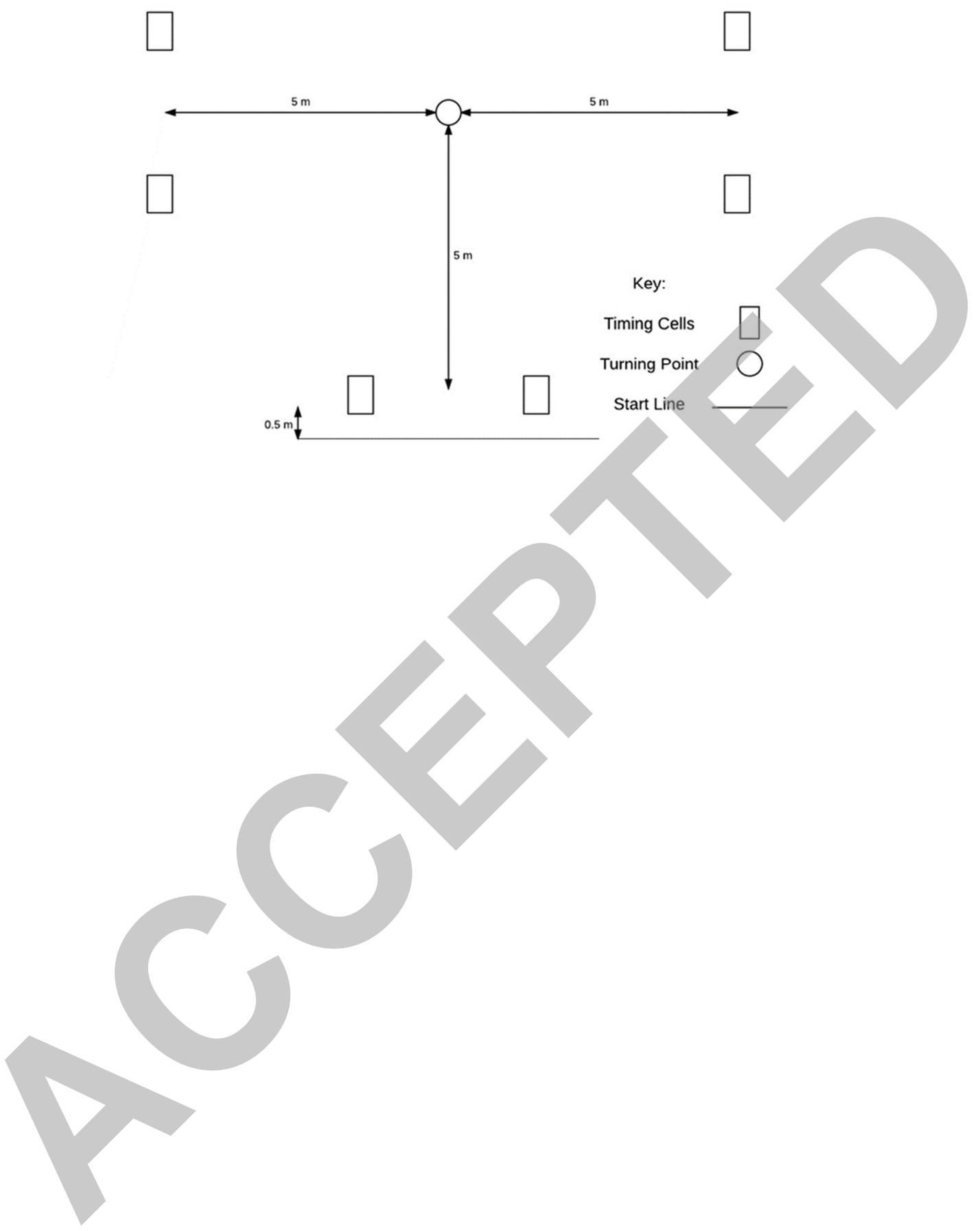




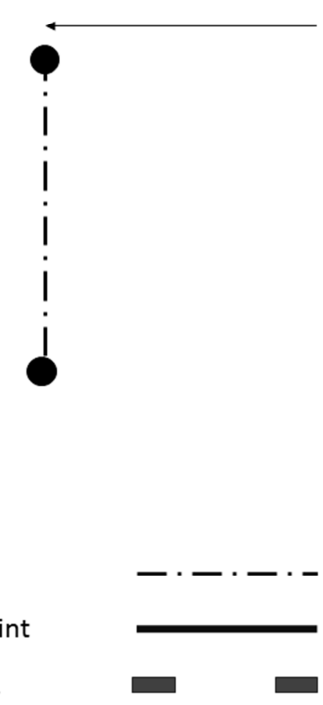

$10 \mathrm{~m}$

Key:

Start point
'Turning' point
Timing cells $5 \mathrm{~m}$

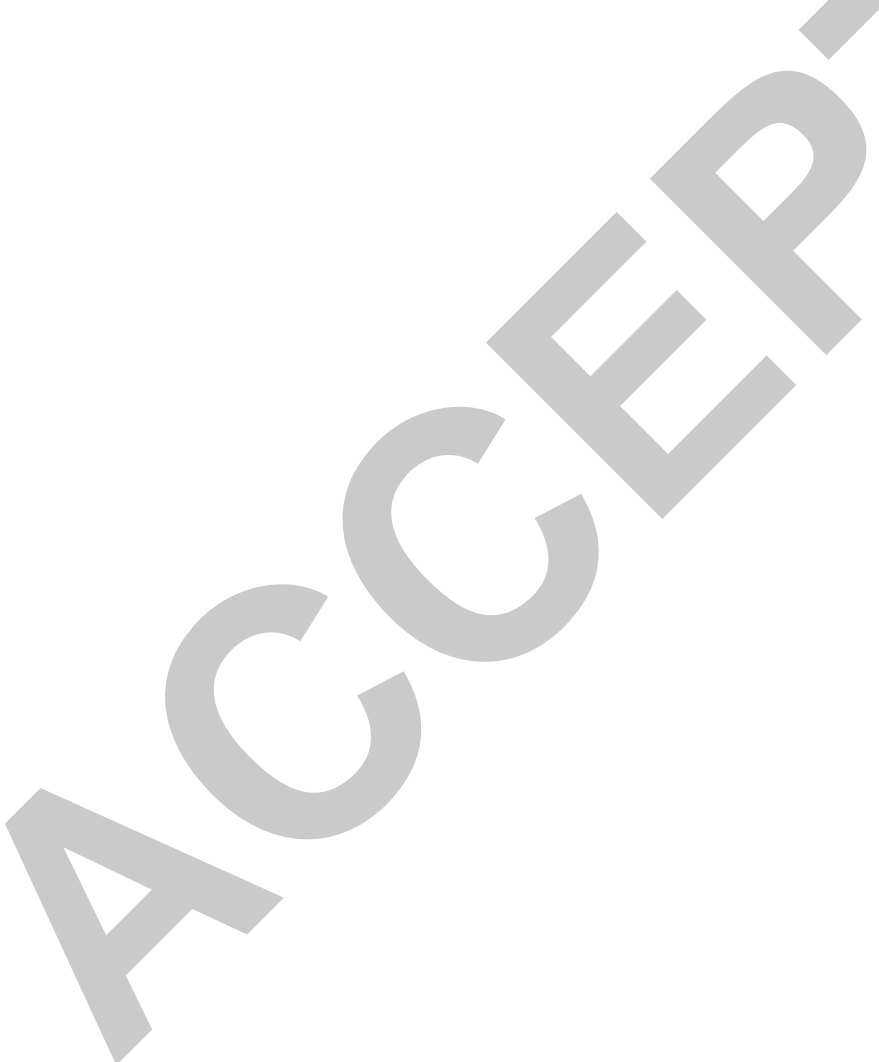




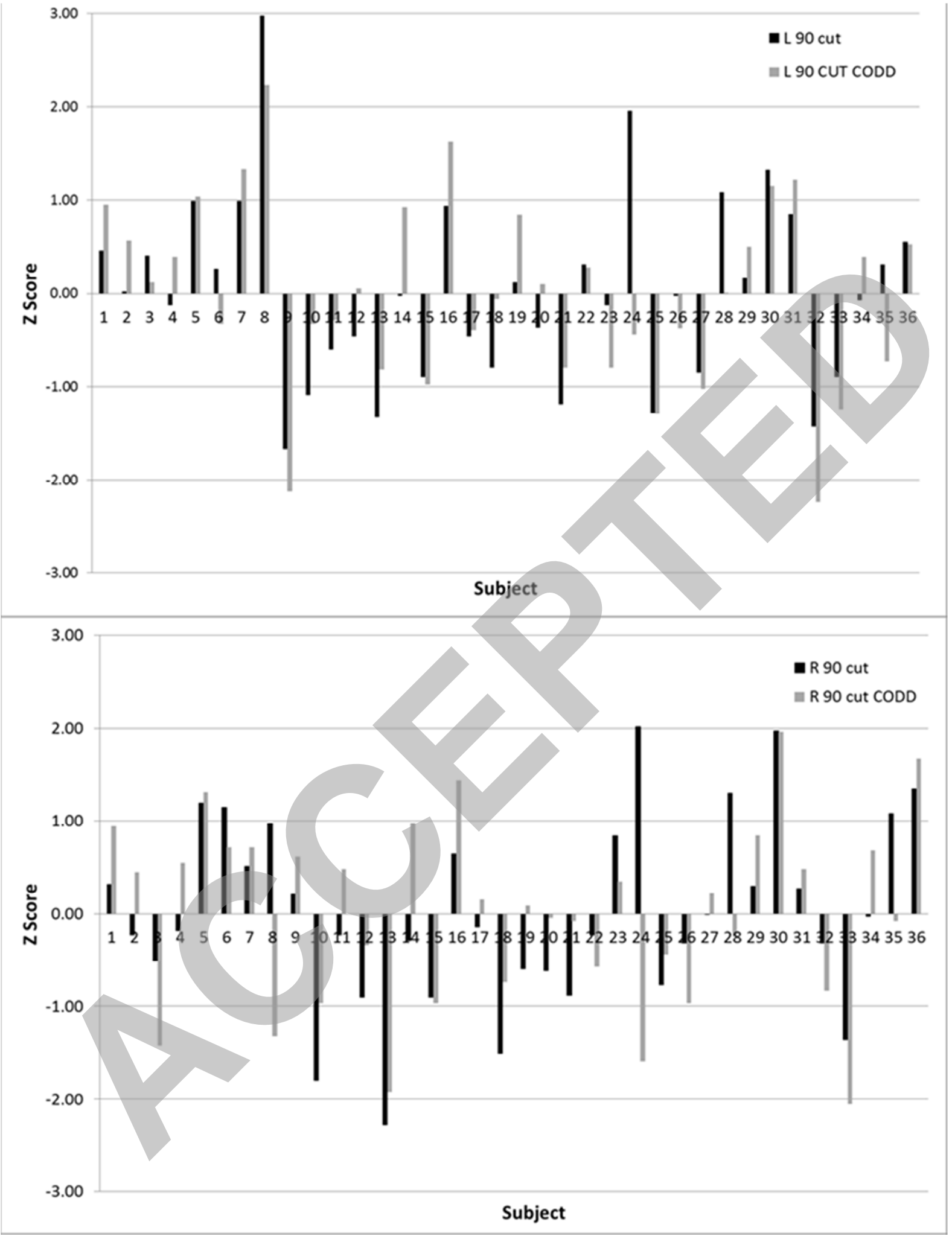

